\section{Incidencia de infarto agudo de miocardio en Chile, 2008-2016}

\author{
CAROLINA NAZZAL ${ }^{1}$, ALEJANDRO LEFIAN ${ }^{2}$, FAUSTINO ALONSO $^{1}$
}

\section{Incidence of acute myocardial infarction in Chile between 2008 and 2016}

Background: There is no recent information on the incidence of acute myocardial infarction (AMI) in Chile. Aim: To describe and evaluate the temporal incidence trend of AMI in Chile between 2008 and 2016. Material and Methods: A time series study. We included all AMI cases (ICD10 =I21) that were registered in Chile between 2008 and 2016 in the national hospital discharge and death databases. Rates were stratified according to sex and age group. We calculated crude and standardized rates (direct method). Time trends were evaluated using Prais-Winsten $(P W)$ regression models. Results: There were 132,784 cases of AMI. The mean age of cases was $67 \pm$ 14 years, 67\% were men. Crude and standardized rates were 84.4 and 73.1 cases per 100,000 inhabitants, respectively. Standardized incidence increased in total population and women, whose PW coefficients were 0.43 (0.01-0.82; $p=0.045)$ and $0.26(0.005-0.47 ; p=0.02)$, respectively. Regarding age, an upward trend was observed in the younger age groups, whose coefficients were $0.20(0.08-0.31 ; p=0.004)$ for cases $<45$ years, $1.31(0.81-1.81 ; p<0.01)$ for cases between 45 and 54 years, and 2.68 (1.31-4.04; $p=0.002)$ for cases between 55 and 64 years. Conclusions: An increase in the number of cases with AMI was observed, especially in younger age groups. This estimation could be useful for planning and evaluating public policies.

(Rev Med Chile 2021; 149: 323-329)

Key words: Chile; Incidence; Myocardial infarction.

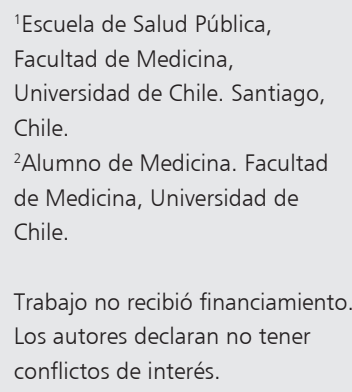

Recibido el 4 de agosto de 2020, aceptado el 13 de enero de 2021

Correspondencia a:

Dra. Carolina Nazzal

Av. Independencia 939,

Independencia. Santiago, Chile. cnazzal@uchile.cl
L

as enfermedades cardiovasculares son la primera causa de muerte en Chile y en el mundo ${ }^{1}$. En Chile, la enfermedad coronaria, y mayoritariamente el infarto agudo de miocardio (IAM), constituyen la primera causa de muerte en hombres y la segunda en mujeres².

Chile está en un proceso de transición epidemiológica avanzada, donde la población envejece progresivamente, con una esperanza de vida a la fecha de 79,73 años (82,09 años en mujeres y 77,18 años en hombres) ${ }^{3}$. Dado este patrón demográfico, la población susceptible de padecer IAM es cada vez mayor, con lo cual se espera que la incidencia de la enfermedad vaya en aumento. Por otra parte, la sobrevida de la enfermedad ha aumentado en la última década, lo que también favorece la incidencia de eventos recurrentes en la población ${ }^{4}$.

Chile cuenta con registros administrativos que permiten realizar estimaciones de incidencia y letalidad, con lo cual, dado que no se cuenta con un registro de incidencia poblacional de IAM, pueden ser utilizados con este fin y acercarse a estimaciones de la incidencia.

El objetivo de este trabajo es estimar la incidencia de IAM en Chile y analizar su tendencia entre los años 2008 y 2016. Esta información puede ser de utilidad en la planificación de las políticas públicas del país. 


\section{Métodos}

Se realizó un estudio de series de tiempo. En el análisis se incluyeron todos los casos de IAM registrados (con y sin supradesnivel del segmento ST) entre enero de 2008 y diciembre de 2016, identificados de las bases de datos de mortalidad y egresos hospitalarios del Departamento de Estadísticas e Información en Salud del Ministerio de Salud (DEIS) para los años mencionados. Se seleccionaron los casos de IAM fatales y no fatales certificados con el código I21 de la décima revisión de la Clasificación Internacional de Enfermedades (CIE 10), considerando como caso un primer episodio o un episodio recurrente de IAM. Se obtuvo información del diagnóstico, edad, sexo, región de residencia, lugar de ocurrencia del evento y desenlace (en caso de hospitalización). Se estimó la incidencia por sexo y por tramos de edad: menores de 45 años, 45 a 54 años, 55 a 64 años, 65 a 74 años y 75 o más años. Además, se estimaron las tasas de incidencia por región ajustadas por edad y sexo.

\section{Análisis estadístico}

Se realizó un análisis descriptivo; la edad fue descrita con la mediana y los percentiles 25 y 75; además, se realizó el análisis de la edad categorizada en grupos, los que fueron descritos con frecuencias absolutas y relativas. Las comparaciones fueron realizadas mediante test de Mann-Whitney para variables continuas y Chi cuadrado para variables categóricas.

Se calculó la tasa de incidencia como el número de casos de IAM (primeros o recurrentes) por 100.000 habitantes. El numerador de la tasa correspondió a la suma de los siguientes eventos: a) hospitalizaciones por IAM en pacientes que egresaron vivos (se considera hospitalización a aquel caso que, al menos, permaneció una noche en el hospital); b) muertes por IAM en pacientes hospitalizados; c) muertes por IAM ocurridas en servicios de urgencia adosados a hospitales, pero que no fueron hospitalizados; d) muertes por IAM extrahospitalarias, es decir, las ocurridas en el domicilio u otro lugar, que incluye servicios de atención primaria de urgencia, postas rurales y consultorios, de acuerdo a la definición utilizada en el certificado de defunción.

El cálculo de tasas crudas de incidencia se realizó utilizando como denominador la pobla- ción total estimada al 30 de junio de cada año, de acuerdo a las proyecciones de población del Instituto Nacional de Estadísticas (INE) de Chile corregidas por el censo del año $2017^{5}$.

Para comparar las tasas por año y evaluar la tendencia del periodo, se realizó la estandarización por método directo ${ }^{6}$, utilizando como población estándar la población mundial de la Organización Mundial de la Salud (OMS) del año 20007.

La tendencia temporal fue evaluada mediante un modelo de regresión de Prais-Winsten (PW), el cual considera la posible autocorrelación de las tasas y, por tanto, se emplea cuando las variables son dependientes del tiempo y los errores están correlacionados ${ }^{8}$. Esta regresión entrega como estimador un coeficiente beta y sus intervalos de confianza de $95 \%$, que muestra el cambio anual de las tasas por 100.000 habitantes. Para estimar el efecto de la edad en los cambios en las cifras de incidencia, se compararon los coeficientes de la regresión PW para las tasas crudas y ajustadas ([coef. tasa cruda-coef. tasa ajustada]/coef. tasa cruda). Finalmente, se estimó el número de casos incidentes para el año 2025.

El nivel de significancia considerado fue de $5 \%$ y los análisis estadísticos fueron realizados con el software STATA $14^{9}$. Se elaboró un mapa colorimétrico con las tasas de incidencia por región (según la división administrativa vigente) utilizando las coberturas oficiales del INE y el software ArcView 3.3.

\section{Resultados}

El número de casos estudiados durante el período 2008-2016 fue 132.784, con un promedio anual de 14.754 casos. Del total de casos, 89.135 fueron hombres $(67,1 \%)$ y 43.649 mujeres $(32,9 \%)$, con una mediana de edad en los hombres de 64 años (p25-p75: 54-74) y en mujeres de 74 años (p25-p75: 62-83). Respecto del total de los casos, la letalidad extrahospitalaria fue $24 \%$ (falleció antes de consultar en un hospital/clínica o servicio de urgencia adosado) y del total de quienes consultaron (76\%), 86,7\% estuvo al menos una noche hospitalizado; finalmente, del total de casos, $59,8 \%$ egresó vivo (Figura 1). El análisis por sexo mostró que los hombres consultaron más que las mujeres a un hospital/clínica $(78,2 \%$ de los hombres vs. $71,4 \%$ de las mujeres $[\mathrm{p}<0,001])$, 


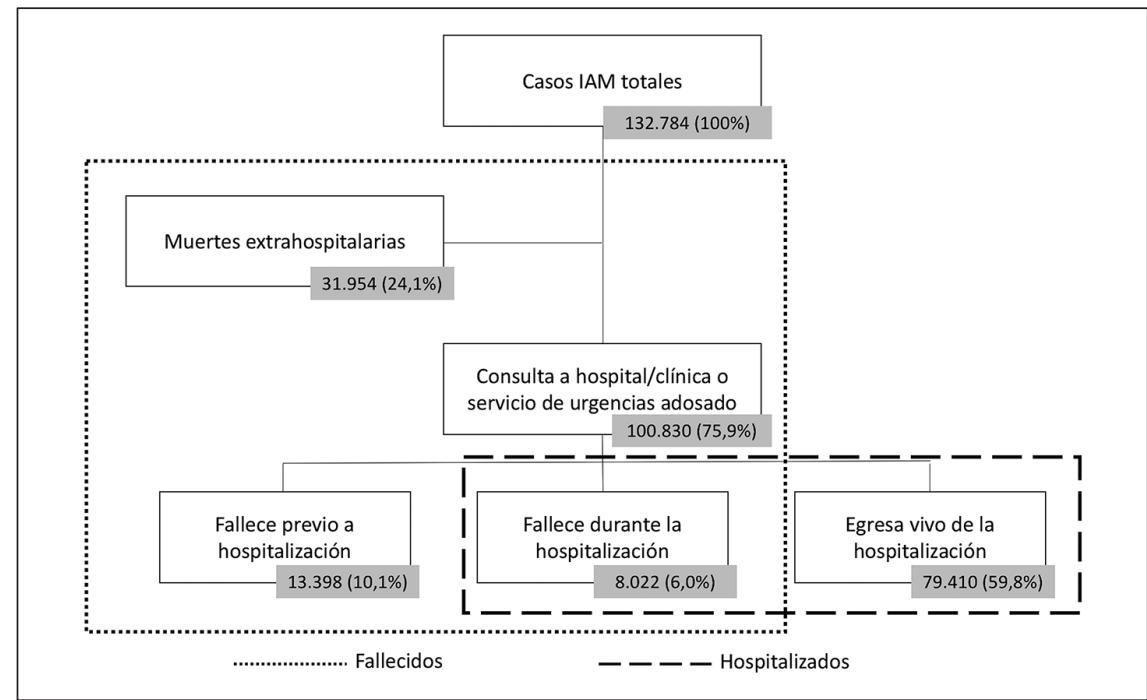

Figura 1. Composición de la población analizada. diferencia que también se presentó al comparar el porcentaje de pacientes que consultaron y fueron hospitalizados $(87,8 \%$ de los hombres vs. $84,1 \%$ de las mujeres $[\mathrm{p}<0,001])$.

Respecto de la edad entre los casos vivos y los fallecidos, estos últimos fueron significativamente de mayor edad (mediana de edad en los vivos 62 años (p25-p75: 53-72) y en los fallecidos 74 años (p25-p75: 63-83 [p < 0,001]).
La tasa cruda promedio de incidencia del período correspondió a 84,3 casos por 100.000 habitantes, siendo mayor en hombres que en mujeres: 114,9 vs. 54,6. La tendencia temporal de las tasas de incidencia según sexo crudas y ajustadas se muestra en la Figura 2. El análisis de tendencia temporal mediante PW para las tasas ajustadas por edad mostró un aumento estadísticamente significativo para la tasa total (coef. PW de 0,42 [IC 95\%:

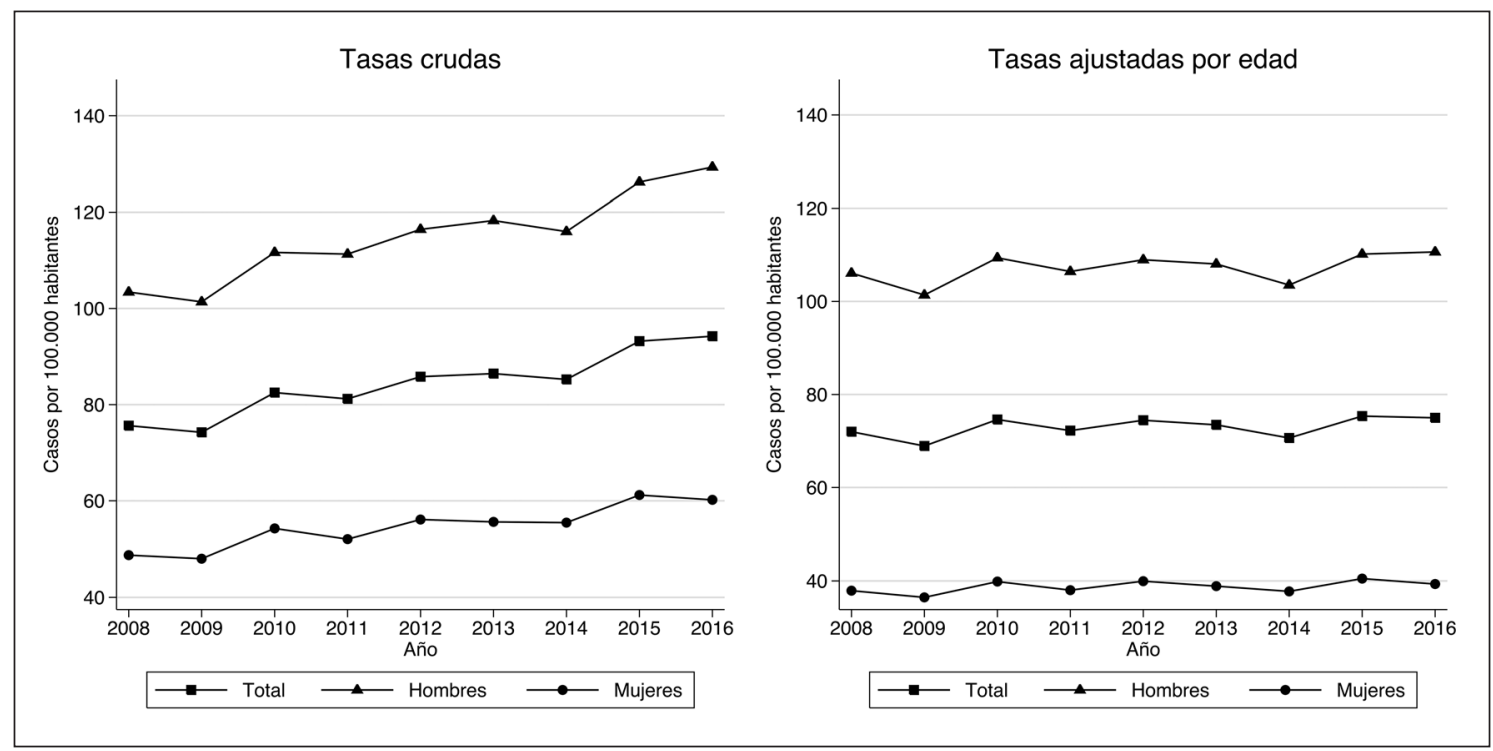

Figura 2. Tasas crudas y ajustadas según sexo, Chile 2008-2016. 


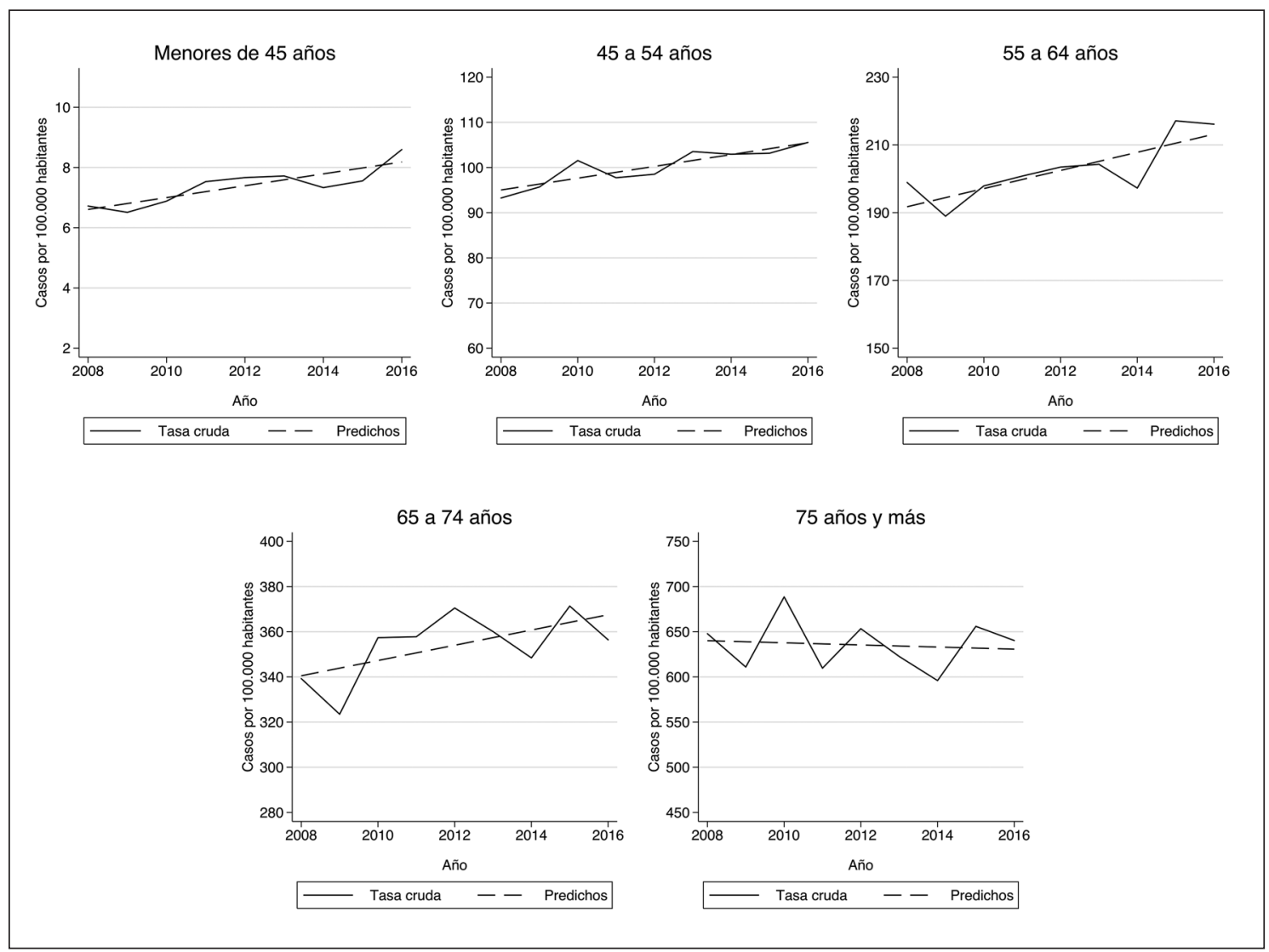

Figura 3. Tasas de incidencia de IAM según grupo etario en Chile 2008-2016. *Valores predichos según modelo de Prais-Winsten.

$0,01$ a 0,$82 ; \mathrm{p}=0,045])$ y para las mujeres (coef. PW de 0,26 [IC 95\%: 0,005 a 0,47; p = 0,022]), sin embargo, este aumento fue marginalmente no significativo en los hombres (coef. PW 0,58 [IC 95\%: -0,04 a 1,19; $\mathrm{p}=0,061]$ ). El porcentaje de la variación explicado por el envejecimiento de la poblacional fue de $82 \%$ en hombres y $83,3 \%$ en mujeres.

La tasa proyectada para el año 2025 es de 115,15 casos/ 100.000 habitantes, lo que equivale a 23.267 personas que presentarían un infarto agudo al miocardio para ese año, de continuar con las tendencias actuales.

En cuanto a la edad, se aprecia un aumento de la incidencia en la medida que aumenta la edad. La incidencia cruda promedio del período para los menores de 45 años fue de 7,39 casos por 100.000 habitantes; en personas de 45 a 54 años: 100,2 . En el grupo de 55 a 64 años la tasa fue
202,8; mientras que entre los 65 a 74 años, 353,9 y en los mayores de 74 años, 636,14. En la Figura 3 se muestra la evolución temporal de las tasas de incidencia cruda del período de acuerdo con el grupo etario y los valores estimados mediante regresión PW. El análisis de tendencia temporal mostró un aumento significativo en la incidencia en los grupos más jóvenes: $<45$ años (coef. 0,20; IC95\%: 0,08 a 0,31: $p=0,004)$; en el grupo de 4554 años (coef 1,31; IC95\%: 0,81 a 1,81:p $<0,0001$ ) $y$ en el de 55-64 años (coef. 2,68; IC95\%: 1,31 a 4,04: $\mathrm{p}=0,002$ ). Los grupos de 65 a 74 años, $y$ el de 75 o más no mostraron una tendencia con significancia estadística (coef. 3,38; IC95\%: -0,21 a 6,96: $\mathrm{p}=0,061)$ y coef. $-1,18$; IC95\%: $-6,56$ a 4,21: $\mathrm{p}=0,622)$.

El análisis espacial (tasas de incidencia regionales ajustadas por sexo y edad) se muestra en la Figura 4. Las regiones Segunda (Antofagasta), 


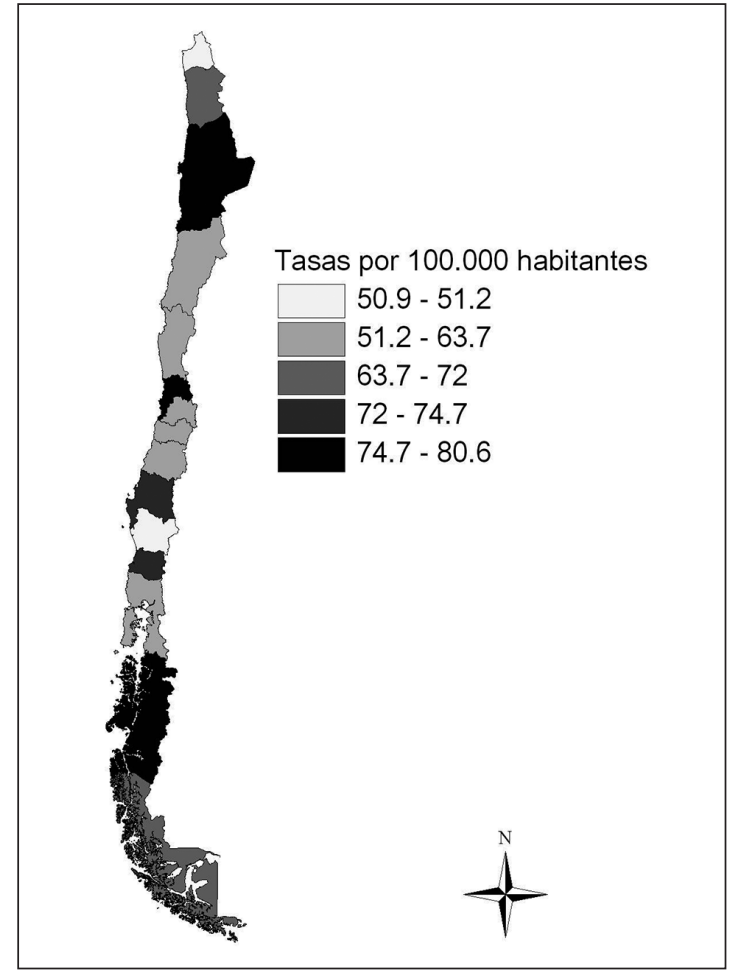

Figura 4. Distribución espacial de los casos de IAM, Chile 2008-2016.

Quinta (Valparaíso) y Undécima (Aysén) mostraron las tasas más altas, mientras que la Decimoquinta (Arica y Parinacota) y la Novena ( $\mathrm{La}$ Araucanía) presentaron las tasas más bajas.

\section{Discusión}

Los resultados muestran que la tasa de incidencia de IAM ha aumentado en Chile entre 2008 y 2016, tanto en términos absolutos como relativos. A nivel internacional se observan distintos escenarios, tanto de aumento, como disminución ${ }^{10,11}$, presentando Chile una situación intermedia con un aumento leve, explicado principalmente por el aumento en mujeres. Sin embargo, la tasa de incidencia sigue siendo mayor en hombres y aumenta progresivamente con la edad. Dado el envejecimiento de la población ${ }^{11,12}$, es esperable un incremento en el número absoluto de casos en las próximas décadas.

Al comparar la evolución de las cifras con lo estimado para la década pasada con la misma metodología ${ }^{4}$, se observó un aumento en las tasas estandarizadas por edad en las mujeres, lo cual es coincidente con otros estudios ${ }^{10,13,14}$. Esto podría, en parte, explicarse por la mejor detección, relacionada con la implementación del sistema de garantías explícitas de salud (GES) en IAM, que garantiza la oportunidad de diagnóstico y tratamiento para toda la población ${ }^{15}$. La letalidad del periodo fue menor a la reportada para los años 2001-2007, tanto para las muertes ocurridas dentro del hospital (8,3\% vs. $6,04 \%)$ como para aquellos que no alcanzaron a consultar $(29,5 \%$ vs. $24,1 \%)^{4}$. Esto último podría deberse a un mejor acceso al sistema de salud y, también, a la mayor conciencia por parte de la población de los síntomas que sugieren un infarto agudo al miocardio. A este respecto, el Ministerio de Salud y la sociedades científicas han realizado campañas para mejorar la percerpción de riesgo y educar sobre cómo actuar frente al IAM. Estas tendencias en letalidad también se han observado en distintos estudios y contextos $^{16,17}$.

Al comparar los resultados del estudio actual con las estimaciones del periodo 2001-2007, encontramos que la edad de presentación se redujo en un año en hombres y en mujeres ${ }^{4}$. Es relevante mencionar que el aumento de las tasas de incidencia está dado por una mayor ocurrencia de casos en los pacientes jóvenes y de edad media, tendencia que ya se había observado en Chile en la década anterior, al igual que en estudios internacionales ${ }^{18,19}$. Esto podría explicarse por el bajo control de los factores de riesgo cardiovascular en la población, como lo muestra la última Encuesta Nacional de Salud (ENS), donde el único factor de riesgo que ha disminuido es el tabaquismo ${ }^{20}$. Adicionalmente, el score de riesgo cardiovascular más utilizado en Chile para establecer la susceptibilidad de presentar un evento cardiovascular y, por ende, indicar acciones preventivas, es el score de Framingham. Este muestra un rendimiento deficiente en poblaciones menores de 40 años, además de no haber sido diseñado para menos de 30 años $^{21,22}$. Lo anterior puede llevar a una falla en la pesquisa de riesgo cardiovascular en poblaciones jóvenes y, por lo tanto, falta de control en esta población. Un segundo factor que podría estar influyendo es el aumento en el consumo de cocaína, reportado por los estudios de drogas en 
población general del SENDA ${ }^{23}$. Otra explicación posible es una mayor pesquisa de enfermedad coronaria, al hacer más extendido el uso de enzimas cardíacas y electrocardiograma, atribuible a la implementación de la guía clínica GES desde 2005, con sus posteriores modificaciones ${ }^{15}$.

En cuanto a la distribución espacial de la inciencia, esta es heterogénea, siendo las regiones de Aysén, Valparaíso y Antofagasta las de mayores tasas, lo que podría explicarse por particularidades regionales relacionadas a exposiciones ambientales $^{24-26}$ y por una mayor prevalencia de factores de riesgo cardiovasculares, como se aprecia en la última $\mathrm{ENS}^{20}$.

Dentro de las limitaciones de este análisis se encuentra el empleo de bases de datos secundarias que pueden presentar errores de codificación en la causa de muerte. Si bien en Chile sobre 99\% de los certificados de defunción son emitidos por médicos, esto no garantiza que la causa de muerte sea registrada correctamente. No hay estudios a nivel nacional que validen la causa de muerte de los certificados de defunción por infarto agudo al miocardio, pero estudios internacionales muestran que la mortalidad por enfermedad coronaria es sobrestimada en aproximadamente $20 \%$ de los certificados de defunción, y que la sobreestimación se reduce a mayor edad ( $>74$ años) y no se presentan variaciones por $\operatorname{sexo}^{27,28}$. Por otra parte, la estimación de la incidencia puede estar afectada por un subdiagnóstico de los pacientes con IAM sin supradesnivel del segmento ST, aunque el empleo de troponinas en la última década ha crecido considerablemente, mejorando el diagnóstico de esta entidad ${ }^{15}$.

Como fortaleza destacamos la representatividad del estudio, porque se han considerado todos los casos de IAM registrados en Chile durante el período y se realizaron análisis por sexo y edad, y a nivel regional.

Esperamos que estos resultados sean de utilidad a las autoridades de salud y la comunidad médica para la implementación y evaluación de las políticas públicas destinadas al control de la cardiopatía isquémica en Chile.

\section{Referencias}

1. World Health Organization. Global Burden of Disease Study 2017. 2017; 1-7.
2. Ministerio de Salud (MINSAL). Departamento de Estadísticas e Información de Salud (DEIS). Indicadores Básicos de Salud Chile. 2016.

3. Departamento de Estadísticas e Información de Salud [Internet]. [citado el 23 de octubre de 2019]. Disponible en: http://www.deis.cl.

4. Nazzal C, Alonso FT. Incidencia y letalidad por infarto agudo del miocardio en Chile: 2001-2007. Rev Med Chile 2011; 139: 1253-60.

5. Instituto Nacional de Estadísticas. Chile. Proyecciones y Estimaciones de Población. Total País CHILE: Proyecciones y Estimaciones de Población. Total País. Inst Nac Estad. 2006; 103.

6. OPS. ASDI/Organización Panamericana de la Salud. XVI Reunión del Sector Salud de Centroamérica y República Dominicana (RESSCAD), Informe Final. Honduras, 2000. Comisión Económica para América Latina y el Caribe. Boletín Epidemiológico [Internet]. 2002; 23 (3): 9-12. Disponible en: http://www.cepal.cl/ publicaciones/poblacion/9/LCG2059/BD64.html

7. Ahmad OB, Boschi-Pinto C, López AD, Murray CJ, Lozano R, Mie I. Age standardization of rate: A new who standard (GPE Discussion Paper Series: No. 31 EIP/ GPE/EBD). World Health Organ. 2001; 31.

8. Durbin J, Watson GS. Corrections to Part I: Testing for Serial Correlation in Least Squares Regression: I. Biometrika 1950; 37(3-4): 409-28.

9. StataCorp. Stata Statistical Software: Release 14. College Station, TX: StataCorp LP. 2015.

10. Sanchis-Gomar F, Pérez-Quilis C, Leischik R, Lucia A. Epidemiology of coronary heart disease and acute coronary syndrome. Ann Transl Med. 2016; 4 (13): 1-12.

11. Yeh RW, Go AS. Rethinking the epidemiology of acute myocardial infarction: Challenges and opportunities. Arch Intern Med. 2010; 170 (9): 759-64.

12. Instituto Nacional de Estadísticas. Estimaciones y proyecciones de la población de Chile 2002-2035. Totales regionales, población urbana y rural. 2019; 25.

13. Reynolds K, Go AS, Leong TK, Boudreau DM, Cassidy-Bushrow AE, Fortmann SP, et al. Trends in Incidence of Hospitalized Acute Myocardial Infarction in the Cardiovascular Research Network (CVRN). Am J Med 2017; 130(3): 317-27.

14. Rosamond WD, Chambless LE, Heiss G, Mosley TH, Coresh J, Whitsel E, et al. Twenty-two-year trends in incidence of myocardial infarction, coronary heart disease mortality, and case fatality in 4 US communities, 1987-2008. Circulation 2012; 125(15): 1848-57.

15. Ministerio de Salud S, Pública. Guía Clínica 2010 Infarto Agudo del Miocardio con Supradesnivel del Segmento ST. $2010 ; 1-44$. 
16. Orozco-Beltrán D, Cooper RS, Gil-Guillen V, Bertomeu-Martínez V, Pita-Fernández S, Durazo-Arvizu R, et al. Trends in Mortality From Myocardial Infarction. A Comparative Study Between Spain and the United States: 1990-2006. Rev Española Cardiol. English Ed. 2012; 65(12): 1079-85.

17. Dégano IR, Salomaa V, Veronesi G, Ferriéres J, Kirchberger I, Laks T, et al. Twenty-five-year trends in myocardial infarction attack and mortality rates, and case-fatality, in six European populations. Heart 2015; 101(17):1413-21.

18. Alzuhairi KS, Søgaard P, Ravkilde J, Gislason G, Køber L, Torp-Pedersen C. Incidence and outcome of first myocardial infarction according to gender and age in Denmark over a 35-year period (1978-2012). Eur Hear J - Qual Care Clin Outcomes. 2015; 1 (2): 72-8.

19. Arora S, Stouffer GA, Kucharska-Newton AM, Qamar A, Vaduganathan M, Pandey A, et al. Twenty Year Trends and Sex Differences in Young Adults Hospitalized with Acute Myocardial Infarction: The ARIC Community Surveillance Study. Circulation 2019; 139(8): 1047-56.

20. Ministerio de Salud (MINSAL). Encuesta Nacional de Salud 2016-2017 Primeros resultados. Departamento de Epidemiología, División Planificación Sanitaria, Subsecretaría de Salud Pública [Internet]. 2017; 61. Available from: http://web.minsal.cl/wp-content/ uploads/2017/11/ENS-2016-17_PRIMEROS-RESULTADOS.pdf.

21. Berry JD, Lloyd-Jones DM, Garside DB, Greenland P. Framingham risk score and prediction of coronary heart disease death in young men. Am Heart J. 2007; 154 (1): 80-86.

22. Hemann BA, Bimson WF, Taylor AJ. The Framingham Risk Score: an appraisal of its benefits and limitations. Am Heart Hosp J. 2007 Spring; 5 (2): 91-6.

23. Gobierno de Chile S. Servicio Nacional para la Prevención y Rehabilitación del Consumo de Drogas y Alcohol. Décimo segundo estudio nacional de drogas en población general de Chile, 2016. 2016.

24. Du Y, Xu X, Chu M, Guo Y, Wang J. Air particulate matter and cardiovascular disease: The epidemiological, biomedical and clinical evidence. J Thorac Dis. 2016; 8 (1): E8-19.

25. Farhadi Z, Abulghasem Gorgi H, Shabaninejad H, Aghajani Delavar M, Torani S. Association between PM2.5 and risk of hospitalization for myocardial infarction: A systematic review and a meta-analysis. BMC Public Health. 2020; 20 (1): 1-12.

26. Moon K, Guallar E, Navas-Acien A. Arsenic exposure and cardiovascular disease: An updated systematic review. Curr Atheroscler Rep. 2012; 14 (6): 542-55.

27. Coady SA, Sorlie PD, Cooper LS, Folsom AR, Rosamond WD, Conwill DE. Validation of death certificate diagnosis for coronary heart disease: The Atherosclerosis Risk in Communities (ARIC) Study. J Clin Epidemiol. 2001; 54 (1): 40-50.

28. Svendsen MT, Boggild H, Skals RK, Mortensen RN, Kragholm K, Hansen SM, et al. Uncertainty in classification of death from fatal myocardial infarction: A nationwide analysis of regional variation in incidence and diagnostic support. PLoS One 2020; 01: 1-15. 